\title{
Violência entre Parceiros Íntimos e as Implicações para a Saúde da Mulher
}

\section{Violence between Intimate Partners and its Implications for Women's Health}

\author{
Lélio Moura Lourenço', Dayane Pereira Costa²
}

\begin{abstract}
Resumo
A violência entre parceiros íntimos é considerada pelos órgãos governamentais como uma das maiores causas de alterações do estado de saúde das mulheres. O presente trabalho investigou por meio de uma revisão sistemática da literatura as consequências da violência doméstica entre parceiros íntimos para a saúde da mulher. Foram selecionados artigos das seguintes bases de dados: PubMed, PsycInfo, Redalyc, SciELO, Pepsic e Bireme. Os trabalhos foram divididos em duas planilhas, uma para Violência Doméstica e outra para Violência entre Parceiros Íntimos. Após, aplicados os critérios de inclusão e exclusão foram incluídos 13 produtos da tabela de VD e sete da tabela de VPI, totalizando uma amostra de 20 artigos para análise. Os resultados sugerem que existe uma significativa relação entre violência entre parceiros íntimos e agravos à saúde da mulher, especialmente à saúde mental. A partir deste estudo, considera-se imprescindível que intervenções que visem minimizar os agravos sejam desenvolvidas.
\end{abstract}

Palavras-chave: Violência entre parceiros íntimos. Saúde da mulher. Saúde mental.

\begin{abstract}
Violence among intimate partners is considered by government agencies as one of the major causes of changes in the health status of women. Through a systematic review of the literature, the present work investigated the consequences of domestic violence among intimate partners for women's health. Articles from the following databases were selected: PubMed, PsycInfo, Redalyc, SciELO, Pepsic and Bireme. The papers were divided into two spreadsheets, one for Domestic Violence and another for Violence between Intimate Partners. After applying the inclusion and exclusion criteria, 13 products of the DV table and 7 of the IPV table were included, totaling a sample of 20 articles for analysis. The results suggest that there is a significant relationship between intimate partner violence and women's health, especially mental health. From this study, it is considered essential that interventions aimed at minimizing these diseases be carried out.
\end{abstract}

\footnotetext{
${ }^{1}$ Universidade Federal de Juiz de Fora, Juiz de Fora, Brasil. Contato: leliomlourenco@gmail.com.

2 Universidade Federal de Juiz de Fora, Juiz de Fora, Brasil. Contato: dayanepcosta@hotmail.com
} 
Keywords: Intimate partner violence. Women's health. Mental health. 


\section{Introdução}

A violência doméstica é uma forma de violência que engloba diferentes subtipos. Dentre estes estão a violência contra crianças, adolescentes, idosos, homens e mulheres. A violência doméstica é caracterizada por agressividade e coação que correspondem aos ataques físicos, sexuais e psicológicos de um indivíduo da família (Albuquerque et al., 2013). Ainda que o termo doméstico faça menção ao contexto das relações familiares como um todo, "violência doméstica" tem sido usada, muitas vezes, para se referir exclusivamente à violência cometida pelo homem contra a mulher (Bhona, Lourenço \& Brum, 2011).

$\mathrm{O}$ estudo aqui apresentado tem como foco a violência entre parceiros íntimos (VPI), visto que a violência contra a mulher se configura como a mais frequente vertente da violência doméstica e, nesse contexto, a maioria dos agressores é composta pelos seus denominados "parceiros íntimos": o esposo, noivo, namorado ou qualquer homem com quem a mulher tenha uma relação íntimo-afetiva. Corroborando essa fala, Oliveira e D’Oliveira (2008) afirmam que a forma mais comum de violência contra a mulher é cometida por parceiros íntimos e estima-se que de $20 \%$ a $50 \%$ das mulheres do mundo sofram violência física e/ou sexual por seus parceiros em algum momento da vida. Presume-se que uma a cada cinco mulheres sofram alguma forma de violência na vida, podendo ocasionar trauma grave ou morte (Brum, Lourenço, Gebara \& Ronzani, 2013).

A violência proveniente de parceiro íntimo (VPI) ocasiona dano físico, sexual ou psicológico, incluindo agressão física, coerção sexual, abuso psicológico e comportamentos controladores (Mendonça \& Ludermir, 2017).

A violência entre parceiros íntimos é multicausal, logo, existem vários determinantes que podem estar envolvidos nesse fenômeno. Dentre esses determinantes estão questões culturais e desigualdades de gênero. Nesse sentido, Lettiere e Nakano (2011) ressaltam que a desigualdade das relações de gênero pode ser a causa e a consequência de as mulheres terem maior risco de serem violentadas em suas relações familiares, destacando que na maioria das vezes o agressor tem sido o próprio cônjuge ou parceiro. No entanto, a posição de agressor não é ocupada somente por homens, as mulheres, em menor proporção, também assumem em alguns momentos o papel de agressora, uma vez que as interações entre os casais são complexas. Ainda que exista uma relação de dominação, ela não é absoluta, existem violências recíprocas entre alguns parceiros (Oliveira \& Almeida, 2013). Contudo, mesmo considerando que a mulher possa ser a perpetradora da violência, na maioria das vezes ela se configura como vítima. Idoko, Ogbe, Jallow e Ocheke (2015), afirmam que mesmo que os homens também possam ser vítimas de violência entre parceiros íntimos, a prevalência das evidências mostra que as mulheres são muito mais propícias a serem as vítimas da violência.

Segundo dados do Sistema de Informação de Agravos de Notificação (Sisan) do Ministério da Saúde, que registra os atendimentos do Sistema Único de Saúde (SUS) no campo das violências, em todas as idades, até os 59 anos, os atendimentos femininos superam os masculinos (Waiselfisz, 2015). A violência contra mulheres se refere ao uso da força física e à ideia de submissão, 
culturalmente impregnada nas relações de gênero, na qual o homem comporta-se como ser dominante $\mathrm{e}$ a mulher um ser inferior $\mathrm{e}$ caracteriza-se por danos à saúde física e mental da vítima (Silva, Valongueiro, Araújo \& Ludermir, 2015).

De acordo com a Organização Panamericana de Saúde (Opas), a violência se tornou um problema de saúde pública devido aos inúmeros agravos à saúde oriundos de situações violentas (Brasil, 2005). Sendo a violência um fenômeno antigo, considera-se que a percepção dos agravos fez com que este fosse considerado um problema de Saúde Pública. A violência tem consequências que afetam a saúde individual e coletiva, bem como os serviços do setor de saúde. As unidades de serviços, antes muito mais orientadas para as enfermidades de origem biomédica, são hoje chamadas para dar respostas às vítimas de lesões e traumas físicos e emocionais (Brasil, 2005).

Segundo dados do Sisan, a cada três vítimas de violência, duas foram mulheres que sofreram violência doméstica e que consequentemente necessitaram de atendimento médico (Waiselfisz, 2015).

Para a Organização Mundial de Saúde (OMS), a violência entre parceiros íntimos é uma das maiores causas de alterações do estado de saúde das mulheres (Brasil, 2005). A saúde da mulher vítima de violência doméstica é afetada de diversas formas e em variados âmbitos e intensidades. As marcas do sofrimento decorrente da vitimização aparecem logo no corpo, na saúde, trazendo sequelas não só físicas, mas também mostrando vários tipos de associação, somatização e prejuízo mental, como ideação suicida, perda de autoestima, depressão, fobias, pesadelos, crises de angústia, psicoses, medo de relações sexuais, dentre outros (Brasil, 2005).

A mulher vítima de violência doméstica tem sua saúde física comprometida e entre as suas consequências, como revela a literatura, estão as cardiopatias, acidentes vasculares, hipertensão arterial, dores crônicas, problemas intestinais e a fibromialgia. Com relação à saúde sexual e reprodutiva, o que se observa são disfunções sexuais, doença inflamatória pélvica, infecções sexualmente transmissíveis e infertilidade. Também podem sofrer de gravidez indesejada, bebês com baixo peso ao nascer, mortes maternas e neonatais (Idoko et al., 2015). As mulheres vítimas também apresentam problemas psicoemocionais. Mulheres que convivem com VPI repetitiva manifestam maiores prejuízos à saúde, em especial à saúde mental. Sintomas como estresse, depressão, ansiedade, distúrbios de sono são recorrentes a essas mulheres (Schraiber, Latorre, França, Segri \& D’Oliveira, 2010).

Diante da dimensão do fenômeno violência doméstica e suas diversas implicações para a saúde da mulher, objetiva-se com este trabalho realizar uma revisão sistemática da literatura para investigar o impacto $\mathrm{e}$ as consequências da violência doméstica entre parceiros íntimos para a saúde da mulher e, em especial, as consequências para a saúde mental das mulheres, além de averiguar o que tem sido publicado sobre o tema em questão. Antes da exposição dos resultados encontrados por esta revisão, é importante a descrição da metodologia utilizada para o estudo. 


\section{Metodologia}

O trabalho em questão teve o intuito de investigar o fenômeno da violência doméstica entre parceiros íntimos e suas consequências para a saúde da mulher. Para tanto, foi realizada uma revisão sistemática da literatura referente à produção científica acerca do tema, nos últimos 10 anos. Para esse fim, foram selecionados artigos das seguintes bases de dados: PubMed, PsycInfo, Redalyc, SciELO, Pepsic e Bireme.

Durante as buscas, foram utilizados os seguintes descritores: "Violência doméstica E Saúde da mulher" e "Violência entre parceiros íntimos E Saúde da mulher" em português, em espanhol foram utilizados os seguintes: "La violencia doméstica Y Salud de la mujer" e "Violencia en la pareja Y Salud de la mujer" e em inglês "Domestic violence AND Woman's health" e "Violence between intimate partners AND Woman's health". Os critérios de seleção dos descritores foram feitos em função das características das bases pesquisadas. Somente foram elencados para análise inicial os trabalhos que compunham o formato de artigo e que foram publicados no período compreendido entre os anos 2007 e 2017.

As buscas nas bases de dados já supracitadas totalizaram 107 resultados. $\mathrm{Na}$ base SciELO foram encontradas 55 publicações, por conseguinte foram encontradas 18 na PsycInfo, 13 na Redalyc, 13 na Bireme, 6 na Pubmed e, por fim, 2 na Pepsic.

Para análise foi utilizado o programa Microsoft Office Excel 2007. Os resultados foram organizados em duas planilhas do Excel, uma para violência doméstica (VD) e outra para violência entre parceiros íntimos (VPI). Nos resultados das buscas, foram obtidos 61 produtos para VD e 46 para VPI. A partir de então, foi feita a análise inicial dos trabalhos coletados por meio da leitura dos títulos e resumos com o objetivo de averiguar quais trabalhos abordavam a temática. Após essa análise inicial, foram excluídos 23 (38\%) artigos da tabela de VD, restando 38 (62\%) para análise e da tabela de VPI foram excluídos $14(31 \%)$ artigos, restando $32(71 \%)$ para análise. Os critérios de inclusão foram os seguintes: (i) abordar o tema violência doméstica entre parceiros íntimos e as consequências para a saúde da mulher, (ii) compor o formato de artigo, (iii) texto completo disponível gratuitamente e (iv) publicado entre os anos 2007 e 2017. Os de exclusão foram: (i) artigos que não abordavam o tema, (ii) material repetido, (iii) artigos que tratavam do tema violência doméstica relacionado a outros assuntos, que não a saúde da mulher. Posteriormente, foi feita a leitura completa dos textos incluídos inicialmente. Feita essa leitura na íntegra e aplicados os critérios de inclusão e exclusão, da tabela de VD mais 25 (66\%) artigos foram excluídos, dentre estes 21 (84\%) por abordar o tema VD relacionado a outros assuntos e quatro $(16 \%)$ por compor material duplicado. Na tabela de VPI mais $23(72 \%)$ trabalhos foram excluídos, 13 (56\%) por abordar o tema VD relacionado a outros assuntos, seis (26\%) por compor material duplicado e $4(17 \%)$ por não estar disponível completo e gratuitamente. Ao final, foram incluídos 13 produtos da tabela de VD e sete da tabela de VPI, totalizando uma amostra de 20 artigos para análise.

Durante a leitura dos artigos, foram utilizadas as seguintes categorias de análise: título, autoria, ano de publicação, área da publicação, base de dados, dados sobre a amostra, os 
instrumentos utilizados, tipos de estudo e os principais resultados. Os resultados obtidos com essas categorias serão descritos a seguir.

\section{Resultados}

A análise dos artigos selecionados sobre Violência Doméstica permitiu a obtenção dos resultados seguidamente descritos, de acordo com as categorias de análise. Considerando a categoria autoria, os autores que mais produziram sobre o tema durante o período pesquisado foram: Nakano, A. e Diniz, N. com três produções cada.

$\mathrm{Na}$ análise de publicações por ano, percebeu-se uma constância no número de artigos no período analisado, variando entre uma e duas publicações, como pode ser observado no gráfico a seguir.

Gráfico 1. Número de publicação em relação ao ano

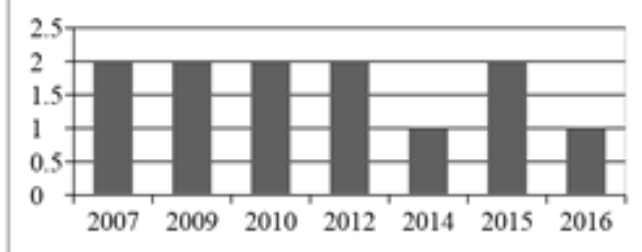

Fonte: Elaborado pelos autores.

A análise da categoria área de publicação demonstrou um predomínio de publicações no campo da Enfermagem, ficando esta composta por oito $(61,5 \%)$ artigos e o restante distribuído entre as outras áreas.

Tabela 1. Número de artigos em relação aos periódicos

\section{Periódicos}

$\mathrm{N}^{\mathbf{o}}$ de artigos

$\begin{array}{ll}\text { Revista Latino Americana de Enfermagem } & 1 \\ \text { Revista Brasileira de Enfermagem } & 2 \\ \text { Acta Paulista de Enfermagem } & 1 \\ \text { Texto \& Contexto Enfermagem } & 2 \\ \text { Psicologia USP } & 1 \\ \text { Saúde em Debate } & 1 \\ \text { Escola Anna Nery Revista de Enfermagem } & 1 \\ \text { Estudos Feministas } & 1 \\ \text { Journal of Human Growth and Development - USP } & 1 \\ \text { International Journal of Clinical and Health } & 1 \\ \text { Psychology } & \end{array}$

Total 
As amostras dos estudos foram compostas, em sua maioria, por mulheres vítimas de violência doméstica por parceiro íntimo. Especificamente, em nove estudos (69\%), os sujeitos da amostra foram mulheres vítimas de violência, entre as quais mulheres que procuraram ajuda em alguma instituição de saúde ou instituições civis.

Em relação ao tipo de instrumento utilizado, pôde-se perceber uma dominante presença de entrevistas, presente em nove estudos (69\%). A metodologia do estudo também foi analisada, sendo encontrados seis $(46 \%)$ estudos de natureza qualitativa, enquanto quatro $(30,7 \%)$ estudos eram de natureza quantitativa.

$\mathrm{Na}$ análise dos resultados dos estudos, optou-se por enfatizar os achados sobre as consequências da violência doméstica para a saúde da mulher, sendo descritos os principais resultados a seguir. Lettiere e Nakano (2011) buscaram investigar o modo de enfrentamento de mulheres vítimas de violência doméstica. Para tanto, entrevistaram 10 mulheres que buscaram ajuda tanto no meio social quanto em serviços de saúde. Em relação às necessidades de saúde, encontraram que dentre as consequências que se mostraram mais evidentes nas falas das mulheres foram as de ordem psíquica, entre elas questões psicossomáticas, emocionais e de autoestima.

Estudo similar analisou as formas de enfrentamento encontradas por nove mulheres vítimas da violência doméstica, no transcorrer e após realizarem a denúncia. Com a descrição das mulheres participantes, encontrou-se que doenças físicas e emocionais estão presentes em suas vidas, queixas como comportamentos agressivos, depressivos, doenças psicossomáticas, insônia, depressão, nervosismo, baixa autoestima e estresse foram relatados (Parente, Nascimento \& Vieira, 2009).

Diniz, Lopes, Rodrigues e Freitas (2007) realizaram um estudo que teve como objetivo geral analisar as lesões corporais por queimaduras em 35 mulheres e sua associação com a violência doméstica. Nessa investigação, também foram encontradas como consequências da violência sequelas psicológicas, tais como falta de concentração, insônia, culpa e problemas mentais, dentre estes depressão, estresse pós-traumático e tendência ao suicídio.

Diniz et al. (2011) também constataram sequelas psicológicas decorrentes da violência. Eles entrevistaram 147 mulheres internadas por aborto provocado numa maternidade pública com o intuito de averiguar a associação entre violência doméstica e o aborto provocado. Eles confirmaram a hipótese e ainda descobriram que essa associação reflete na saúde mental das mulheres, pois ocasiona sintomas do transtorno de estresse pós-traumático. Além disso, descobriram que a violência doméstica durante a gravidez pode causar estresse, depressão e consumo de álcool e drogas.

Gomes et al. (2014), do mesmo modo, entrevistaram 52 profissionais que atuam em Unidades de Saúde da Família visando averiguar a importância referida ao psicólogo em situações de violência conjugal. Os relatos encontrados dizem que as mulheres em vivência de violência conjugal necessitam de apoio psicológico em decorrência de problemas emocionais, como baixa autoestima.

Em uma pesquisa realizada por Silva, Valongueiro, Araújo e Ludermir (2015), foram 
entrevistadas 406 mulheres com o intuito de investigar os agravos à saúde em decorrência da violência doméstica. Entre as principais consequências relatadas estavam: cefaleia, náuseas, sentimento de insegurança, estresse, depressão, dificuldade com novos relacionamentos, tonturas, picos hipertensivos, desconforto na coluna cervical e sono prejudicado.

Díez et al. (2009) executaram uma investigação semelhante que analisou a relação entre violência doméstica e a saúde física e psicológica por meio de entrevistas com 333 mulheres. Nessa pesquisa, as consequências descobertas pelos autores foram enxaquecas, dores de cabeça, problemas respiratórios, dor abdominal, falta de apetite, ingestão de álcool, ingestão de antidepressivos, hipersonia, dificuldades de concentração e de tomadas de decisão.
Com relação ao restante dos estudos analisados, observou-se que os achados sobre as consequências da violência para a vida da mulher foram: marcas físicas e anulação do sujeito, aborto provocado, depressão, sequelas físicas, consequências emocionais, como o medo e agravos a qualidade de vida das mulheres vitimizadas em diversos aspectos (Gomes et al., 2012; Santi, Nakano \& Lettitere, 2010; Oliveira, Viegas, Santos, Silveira \& Elias, 2015; Lucena et al., 2016).

Posteriormente, foram examinados, segundo as categorias de análise, todos os artigos selecionados sobre Violência entre Parceiros Íntimos, e os resultados encontrados serão descritos a seguir.

Em relação à autoria, constatou-se que apenas uma autora apareceu em mais de um artigo, Ludermir, A. com duas publicações. $\mathrm{Na}$ categoria ano de publicação, observa-se que o ano de 2014 destacou-se com três publicações.

Gráfico 2. Número de publicações em relação ao ano

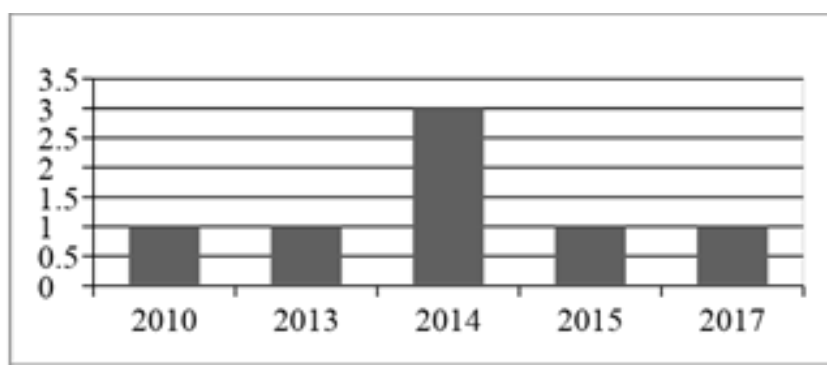

Fonte: Elaborado pelos autores.

Outra categoria de análise foi a área de publicação, ficando os artigos distribuídos nos campos da Enfermagem com dois produtos, Saúde Pública também com dois produtos e a Medicina que, por sua vez, apresentou o maior número, ficando com três produtos. Em relação às revistas, observou-se uma divisão quase equânime entre os artigos analisados. Os periódicos podem ser visualizados na tabela a seguir. 
Tabela 2. Número de artigos em relação aos periódicos

\begin{tabular}{lc}
\hline \multicolumn{1}{c}{ Periódicos } & $\mathbf{N}^{\circ}$ de artigos \\
Revista Brasileira de Enfermagem & 1 \\
Acta Paulista de Enfermagem & 1 \\
Revista de Saúde Pública & 2 \\
Revista Baiana de Saúde Pública & 1 \\
$\begin{array}{l}\text { Revista Brasileira Epidemiol } \\
\text { Caderno de Saúde Pública do Rio de }\end{array}$ & 1 \\
Janeiro & 1 \\
Total & \\
\hline
\end{tabular}

Fonte: Elaborada pelos autores.

Sobre a amostra, em seis $(85,7 \%)$ estudos ela foi constituída por mulheres, entre elas mulheres cadastradas no Programa Saúde da Família e que realizaram registro da violência na Delegacia de Polícia para a Mulher. E, em um (14\%) estudo, a amostra foi composta por outros estudos.

Com relação aos instrumentos, os questionários e entrevistas apareceram três vezes cada. No que se refere à metodologia dos estudos, a maioria expressiva dos artigos analisados apresentou metodologia quantitativa. Os principais resultados estão descritos na sequência.

Schraiber, Barros e Castilho (2010), investigaram a associação entre violência por parceiro íntimo e uso de serviços de saúde, a amostra foi composta por 18 serviços de saúde e 2.674 mulheres usuárias. Agravos como os psicoemocionais, ginecológicos, gastrintestinais e dores no corpo, estiveram muito presentes nos prontuários e mostraram-se associados com VPI. Os autores concluíram que mulheres que convivem com VPI repetitiva apresentam maior frequência de uso de serviços de saúde e de problemas de saúde, em especial de saúde mental.

Outro estudo objetivou avaliar se a violência física entre parceiros íntimos interfere no estado nutricional de mulheres adultas. A amostra foi composta por 625 mulheres e os resultados indicaram que a presença da violência física entre parceiros íntimos associou-se negativamente ao IMC em mulheres. Os autores também inferiram que o estresse crônico presente no dia a dia de uma mulher pobre que vivencia uma situação de violência levaria a distúrbios do sono, abuso de álcool, drogas, tabagismo e uma redução do consumo alimentar (Ferreira et al., 2015).

Mendonça e Ludermir (2017), por sua vez, investigaram a associação da violência por parceiros íntimos relatada contra 390 mulheres nos últimos 12 meses e últimos sete anos com a incidência dos transtornos mentais comuns. Os transtornos mentais mantiveram-se relacionados à violência psicológica, e quando esta esteve combinada com violência física ou sexual, o risco dos transtornos mentais comuns foi ainda mais alto.

Estudo similar foi realizado com o intuito de investigar a associação entre transtornos mentais comuns e violência por parceiro íntimo durante a gravidez. Foram entrevistadas 1.120 mulheres grávidas. A prevalência de transtornos mentais comuns foi 
$71 \%$ entre as mulheres que declararam todas as formas de violência e 33,8\% entre as que não declararam violência por parceiro íntimo. Corroborando o estudo anterior, quando a violência psicológica esteve associada com violência física ou sexual, o risco dos transtornos mentais comuns foi ainda maior (Ludermir, Valongueiro \& Araújo, 2014).

Em uma análise sobre as consequências da violência contra a mulher praticada pelo parceiro, foram entrevistadas 16 mulheres. De acordo com os resultados encontrados, as consequências da violência contra a mulher foram: distúrbios do sono, alimentação inadequada, falta de energia, dores pelo corpo, hematomas, escoriações, síndrome do pânico, tristeza, solidão e baixa autoestima, que determinaram danos psicoemocionais e físicos (Netto, Moura, Queiroz, Tyrrell \& Bravo, 2014). Após analisar separadamente os produtos das tabelas de VD e VPI, realizou-se a análise conjunta das categorias de todos os artigos selecionados. Constatou-se que os autores que mais produziram sobre o tema durante o período pesquisado foram: Nakano, A. e Diniz, N., com três artigos cada.

No que se refere ao ano de publicação dos presentes estudos, o ano de 2014 destacou-se com quatro artigos e, em seguida, os anos de 2010 e 2015, com três artigos cada, como pode ser constatado no gráfico a seguir.

Gráfico 3. Número de publicações em relação ao ano

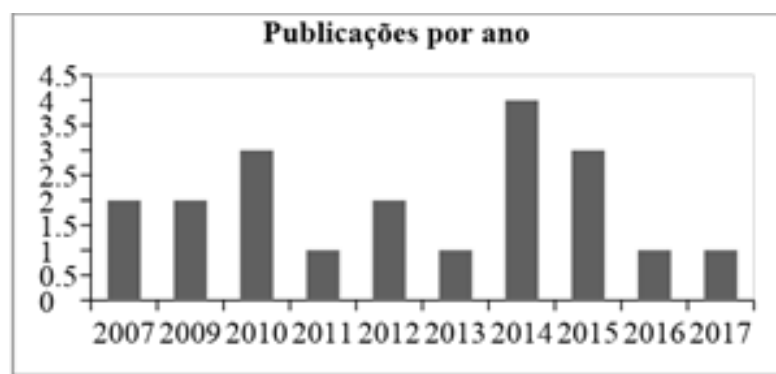

Fonte: Elaborado pelos autores.

Os artigos analisados se originaram de diversos campos do saber, entretanto, houve um predomínio de produções sobre o tema na área da Enfermagem, sendo publicados nessa área 11 artigos (55\%), o que pode ser observado na Tabela 3. 
Tabela 3. Número de artigos em relação à área de publicação

\begin{tabular}{lc}
\hline \multicolumn{1}{c}{ Área } & $\mathbf{N}^{\circ}$ de artigos \\
Enfermagem & 11 \\
Medicina & 3 \\
Saúde Pública & 3 \\
Feminismo & 1 \\
Desenvolvimento & 1 \\
Humano & \\
Psicologia & 1 \\
Total & $\mathbf{2 0}$ \\
\hline
\end{tabular}

Fonte: Elaborada pelos autores.

Os periódicos de publicação analisados número de trabalhos, conforme observado na não apresentaram diferenças expressivas no Tabela 4.

Tabela 4. Número de artigos em relação aos periódicos de publicação

\begin{tabular}{lc}
\hline \multicolumn{1}{c}{ Periódicos } & $\begin{array}{c}\mathbf{N}^{\circ} \mathbf{d e} \\
\text { artigos }\end{array}$ \\
Revista Brasileira de Enfermagem & 3 \\
Acta Paulista de Enfermagem & 2 \\
Texto \& Contexto Enfermagem & 2 \\
Journal of Human Growth and & 2 \\
$\begin{array}{l}\text { Development } \\
\text { Revista de Saúde Pública }\end{array}$ & 2 \\
Caderno de Saúde Pública do Rio de & 1 \\
Janeiro & 1 \\
Revista Brasileira Epidemiol & 1 \\
Revista Baiana de Saúde Pública & 1 \\
Journal of Clinical and Health Psychology & 1 \\
Estudos Feministas & 1 \\
Escola Anna Nery Revista de Enfermagem & 1 \\
Saúde em Debate & 1 \\
Psicologia USP & 1 \\
Revista Latino-Americana de Enfermagem & \\
Total & $\mathbf{2 0}$ \\
\hline
\end{tabular}

Fonte: Elaborada pelos autores.

A amostra de quase a totalidade dos estudos constituiu-se por mulheres, sendo que, em 15 publicações (75\%), as participantes da pesquisa foram mulheres vítimas de violência doméstica por parceiros íntimos que procuraram auxílio em algum serviço de saúde ou instituição para denúncia.
Em relação aos instrumentos utilizados pelos estudos, houve um número expressivo de pesquisas que fizeram uso de entrevista (12 artigos, 60\%) e três estudos que utilizaram questionários (15\%). Quanto à metodologia utilizada, verificou-se que oito $(40 \%)$ estudos 
utilizaram metodologia qualitativa e oito $(40 \%)$ fizeram uso da metodologia quantitativa.

De acordo com os resultados dos produtos, as consequências mais frequentes da violência doméstica entre parceiros íntimos para a saúde da mulher são de ordem mental ou psicológica, tais quais Síndrome do pânico, tristeza, solidão e baixa autoestima (Neto et al., 2014); sintomas do transtorno de estresse póstraumático, estresse, depressão e tendência ao suicídio (Diniz et al., 2011; Diniz, 2007); consequências emocionais como o medo (Oliveira et al., 2015); comportamentos agressivos, depressivos, doenças psicossomáticas e estresse (Parente et al., 2009); sentimentos de insegurança, estresse, depressão e dificuldade com novos relacionamentos (Silva et al., 2015). Outros transtornos mentais comuns também foram encontrados em associação com a violência (Ludermir et al., 2014; Mendonça \& Ludermir, 2017).

Contudo, as consequências da violência contra as mulheres não se limitam à ordem psíquica, também se apresentam como sequelas físicas. Entre os agravos de ordem física estão: distúrbios do sono, alimentação inadequada, falta de energia, dores pelo corpo, hematomas e escoriações (Neto et al., 2014); abuso de álcool, drogas, tabagismo e uma redução do consumo alimentar (Ferreira et al., 2015); problemas ginecológicos, gastrintestinais e dores no corpo (Schraiber et al., 2010); paralisia devido a disparo de armas de fogo e espancamento e abortos provocados por socos na barriga (Oliveira et al., 2015); enxaquecas, dores de cabeça, problemas respiratórios, dor abdominal, falta de apetite, ingestão de álcool, ingestão de antidepressivos e hipersonia (Díez et al., 2009); E, por fim, cefaleia, náuseas, tonturas e picos hipertensivos (Silva et al., 2015).

\section{Discussão}

Com base nos resultados obtidos pela análise dos produtos da amostra, percebeu-se que o número de pesquisas sobre Violência Doméstica entre Parceiros Íntimos e as Consequências para a Saúde da Mulher não tem aumentado ao longo do tempo, a despeito de ser um tema de grande relevância para a sociedade. A relevância da crescente investigação na área se dá, pois, por provocar problemas à saúde física e mental e comprometer o bem-estar das vítimas, daí ser a violência contra as mulheres uma importante questão social e de saúde pública (Diniz et al., 2007). O que foi observado é que existem muitos trabalhos relacionando VD ou VPI a outras áreas temáticas como: a visão dos profissionais de saúde; medidas tomadas pelas Unidades de Saúde; predominância da violência; tipos de violência; fatores associados, entre outros. O mais raro é encontrar trabalhos que abarquem, além da investigação do tema em questão, formas de intervenção, ou seja, questões práticas, pois o argumento de que a violência provoca agravos já é bem conhecido, mas propostas que minimizem suas consequências não são tão difundidas. Logo, investigar esse fenômeno tão frequente é de suma importância, pois os achados das investigações podem servir de base para intervenções eficientes que combatam as consequências advindas da violência contra as mulheres.

Em relação à área de publicação, constatou-se uma predominância de artigos na área da saúde, com destaque para a Enfermagem. 
Nesse sentido, ainda foi possível observar que o maior número de publicações esteve entre as revistas da área da saúde, principalmente da Enfermagem, o que possibilita perceber maior interesse dessa área pelo tema. Isso pode ser explicado pela Organização Pan-Americana de Saúde, que afirma que a interface entre atos violentos e a saúde acontece, pois o setor da saúde é um ponto de cruzamento, tanto por ser o local para onde convergem os casos resultantes da violência como pela pressão que suas vítimas exercem sobre os serviços de urgência, especializados, de reabilitação física e psicológica e de assistência social (Lettiere \& Nakano, 2011). O campo da Psicologia, por sua vez, apresentou somente um artigo, no entanto, considera-se importante que mais estudos sobre o tema sejam desenvolvidos nesse campo, pois, como afirmam Schraiber et al. (2010), a violência por parceiros íntimos acarreta agravos à saúde da mulher, em especial à saúde mental delas. Esse fato pode estar relacionado ao elevado número de casos de violência psicológica, pois, como aponta a literatura, a violência mais prevalente é a psicológica, seguida da violência física e da violência sexual. Com frequência, esse tipo de violência não é percebido pelas vítimas como agressão, o que pode fazer com que muitas mulheres convivam com ele e aceitem suas consequências, logo, a Psicologia tem um campo de atuação vasto nessa área, em que intervenções que visem tanto a conscientização das vítimas quanto à minimização das sequelas devem ser implementadas. Pelo exposto, percebe-se a relevância da etapa de conscientização, principalmente quando se trata da violência psicológica, no entanto, nas buscas não foram encontrados trabalhos que descrevessem alguma intervenção desse tipo.

A partir dos dados extraídos dos resultados dos artigos, foi possível averiguar os principais agravos acarretados à saúde da mulher em decorrência da violência. Com base nos resultados, constatou-se que grande parcela dos estudos, especificamente 15, mencionou consequências de ordem psíquica. Corroborando esse dado, um estudo realizado com mulheres sobre suas experiências psíquicas apontou que vivenciar a violência nas relações familiares, em especial com o companheiro, promove sofrimento psíquico (Vieira et al., 2014). Logo se vê a importância do olhar atento e crítico do psicólogo sobre esse fenômeno.

Diante desse quadro, ressalta-se a importância de profissionais da área de saúde mental, inclusive de psicólogos, em instituições que atendem mulheres vítimas de violência. No âmbito da Atenção Primária à Saúde (APS), recomenda-se na Estratégia Saúde da Família (ESF) a presença de, pelo menos, um profissional da área de saúde mental, pois, além de intervir sobre as sequelas psicológicas, a atuação dos psicólogos viabiliza o fortalecimento da mulher no sentido de traçar saídas para a relação conjugal violenta (Gomes et al., 2014). Com isso, nota-se novamente a importância da presença do psicólogo em instituições que atendem vítimas desse fenômeno e, , de que suas práticas visem mais do que atenuar as sequelas psicológicas, mas também medidas que visem à conscientização e ao fortalecimento da mulher.

Contudo, outros profissionais que atuam com mulheres vítimas de violência também devem estar atentos às consequências advindas do fenômeno, inclusive, consequências 
psicológicas e emocionais, para que as mulheres possam ser encaminhadas para receber os cuidados necessários, os agravos possam ser tratados e a violência combatida. Mas o que se observa na prática é que na maioria dos casos as necessidades sociais e de saúde são apenas tangenciados pelos profissionais (Lettiere \& Nakano, 2011). Com isso, é importante um melhor preparo por parte desses profissionais e também o apoio do profissional de psicologia na equipe de atendimento à mulher vítima de violência, para um melhor reconhecimento e reparo de problemas que estão além do visível. Portanto, reconhece-se que entre as atividades que o psicólogo pode desenvolver, com a equipe de profissionais que atuam com as vítimas, estão programas psicoeducativos para melhor preparar esses profissionais para um efetivo reconhecimento das necessidades das mulheres para que, então, elas possam ser devidamente encaminhadas para receber o suporte necessário.

O que se observa com as consequências psicológicas é que muitas delas, tais como tristeza, solidão, baixa autoestima, tendência ao suicídio, estresse e medo se configuram como sintomas de transtornos mentais comuns como a Depressão e ao Transtorno de Estresse Pós-Traumático, também citados em vários dos produtos como agravos decorrentes da violência (Diniz, 2007; Diniz et al., 2011).

Concernente aos agravos físicos, uma questão observada foi a associação destes com alguns problemas psicológicos. Por exemplo, a insônia, os problemas de alimentação e a falta de energia, que além de serem problemas físicos, também se apresentam como sintomas da Depressão (Powell, Abreu, Oliveira \& Sudak, 2008). O que pode ser observado com essa análise é que a violência psicológica não provoca apenas consequências psicológicas e que a violência física não acarreta apenas problemas físicos. Portanto, deve-se ter uma visão holística da vítima acerca das consequências da violência, para que a atuação do profissional seja efetiva. Logo, o trabalho multi e até interprofissional adquire bastante relevância nesse contexto.

Já outras consequências físicas correspondem diretamente à violência física, que para Albuquerque et al. (2013), se caracteriza por lesões corporais causadas por tapas, socos, chutes, imobilizações, espancamentos, queimaduras de genitália e mamas, estrangulamento e ferimentos com armas brancas, sendo os golpes direcionados para o rosto, braços e pernas da vítima. Logo, esses atos de violência ocasionam dores pelo corpo, hematomas, escoriações, dores de cabeça, tonturas, entre outros. Alguns chegam até a provocar problemas ginecológicos e reprodutivos mais sérios, como o aborto.

Ficou evidente, por meio da análise, o elevado número de comprometimentos psicológicos e emocionais presentes nas mulheres vítimas de violência, com isso se torna relevante discorrer um pouco acerca do assunto.

\section{Saúde mental: depressão e Tept}

As disfunções que estiveram entre as mais citadas pelos trabalhos foram Depressão e Transtorno de Estresse Pós-Traumático (Tept). Além disso, vários estudos têm apontado a comorbidade entre Tept e Depressão em mulheres vítimas de VPI (Felippe, Jesus, Silva, Lourenço \& Grincenkov, 2016).

Em seis estudos, a Depressão foi citada como uma consequência da violência contra a 
mulher, e em outros seis estudos nos quais o transtorno não foi referido, alguns dos seus sintomas foram evidenciados. Com isso, percebese uma significativa prevalência de sintomatologias depressivas entre mulheres vítimas de VPI, o que merece mais atenção dos profissionais de saúde mental e coloca o psicólogo com a responsabilidade de atuar nesse meio, pois sendo um profissional especializado nessas questões, pode oferecer um suporte adequado para as vítimas.

Com relação ao Tept, não houve significativa presença desse transtorno nos estudos, sendo citado em apenas dois trabalhos. Entretanto, alguns sintomas apareceram em oito artigos, logo, percebe-se grande relação entre o transtorno e a violência. E como afirmam Felippe et al. (2016), devido à relevância e gravidade do assunto, surge como algo novo associar o Tept à violência contra a mulher praticada por parceiros íntimos. O que evidencia mais uma vez que a violência pode, além de ocasionar sintomas, tomar a configuração de transtornos mentais que devem ser tratados para que não tomem grandes proporções.

O estudo de Diniz et al. (2007) mostrou que mais de $50 \%$ das mulheres que sofreram violência relataram reviver o trauma, sofrer de insônia, de culpa, evitando associações com um fato que diga respeito ao evento traumático. Com isso, os mesmos autores inferem que a violência doméstica faz com que as mulheres desenvolvam o Transtorno do Estresse Pós-Traumático, sendo necessário que tal transtorno seja encarado como um problema de saúde.

\section{Considerações finais}

Foi possível com este estudo, concluir que são inúmeras as consequências da violência doméstica entre parceiros íntimos para a saúde da mulher. Do mesmo modo, pôde-se perceber que o problema da VD/VPI é bastante discutido e investigado no meio científico, contudo, suas consequências para a saúde da mulher não tem despertado igual interesse, pois não houve tantos estudos que averiguaram diretamente as duas questões quanto estudos que investigaram a violência isoladamente ou associada a outros temas, o que se torna um paradoxo mediante a relevância do tema.

É importante frisar que as pesquisas estão sendo feitas e a qualidade destas se faz presente na maioria das pesquisas em voga, no entanto, a intensificação dessas pesquisas pode e deve promover melhora não só na descoberta e na investigação de processos fundamentais no estudo da violência contra a mulher e suas consequências na saúde, como também no sentido de uma intervenção no ambiente familiar, no ambiente do casal e, por que não, na Saúde Pública, que vise minimizar os agravos ocorridos e promover o bem-estar.

Da mesma forma, é importante que os profissionais de saúde, inclusive da Psicologia, visto a grande parcela de comprometimentos psicológicos ocasionados pela violência, estejam preparados para acolher e oferecer atendimentos efetivos a essas mulheres, para que os danos sejam devidamente tratados e, dessa forma, as mulheres sejam estimuladas a procurarem ajuda no enfrentamento dessas situações, além de proporcionar um ambiente de acolhimento e compreensão. Destaca-se que os serviços 
oferecidos às vítimas do fenômeno sejam realizados por equipe multidisciplinar, visto a interação dos diversos tipos de sintomas e a implicação de variados fatores envolvidos no caso (emocionais, econômicos, sociais e culturais). Além disso, é imprescindível que as intervenções sejam direcionadas tanto para as consequências que podem acometer a mulher, dessa maneira com um caráter preventivo, quanto para as consequências que já ocorreram, visando ao alívio dos sintomas e à promoção da qualidade de vida.

\section{Referências}

Albuquerque, J. B. C., Cesar, E. S. R., Silva, V. C. L., Espíndola, L. L., Azevedo, E. B., \& Ferreira Filha, M. O. (2013). Violência doméstica: características sociodemográficas de mulheres cadastradas em uma Unidade de Saúde da Família. Revista Eletrônica de Enfermagem, 15(2), 382-90.

Bhona, F. M. C., Lourenço, L. M., \& Brum, C. R. S. (2011). Violência doméstica: um estudo bibliométrico. Arquivos Brasileiros de Psicologia, 63(1), 1-110.

Bonfim, E. G., Lopes, M. J. M., \& Peretto, M. (2010). Os registros profissionais do atendimento pré-natal e a (in)visibilidade da violência doméstica contra a mulher. Escola Anna Nery Revista Enfermagem, 14(1) 97-104.

Brasil. (2005). Impacto da violência na saúde dos brasileiros. Brasília: Editora MS.

Brum, C. R. S., Lourenço, L. M., Gebara, C. F. P., \& Ronzani, T. M. (2013). Violência doméstica e crenças: intervenção com profissionais da atenção primária de saúde. Psicologia em Pesquisa, 7(2), 242-250.

Díez, S. U., Escutia, C. V., Pacheco, B. N., Martinez, M. S., Caracena, N. V., \& Contreras, A. O. (2009). Prevalence of intimate partner violence and its relationship to physical and psychological health indicators. Journal of Clinical and Health Psychology, 9(3), 411-427.

Diniz, N. M. F., Gesteira, S. M. A., Lopes, R, L. M., Mota, R. S., Pérez, B. A. G., \& Gomes, N. P. (2011). Aborto provocado e violência doméstica entre mulheres atendidas em uma maternidade pública de Salvador-BA. Revista Brasileira de Enfermagem, 64(6), 10101015.

Diniz, N. M. F., Lopes, R. L. M., Rodrigues, A. D., \& Freitas, D. S. (2007). Mulheres queimadas pelos maridos ou companheiros. Acta Paulista Enfermagem, 20(3), 321-325.

Felippe, A. M., Jesus, S. R., Silva, Y. V., Lourenço, L. M., \& Grincenkov, F. S. R. (2016). Violência praticada pelo parceiro íntimo e o transtorno de estresse pós-traumático. Revista Psique, 1(2), 95-111.

Ferreira, M. F., Moraes, C. L., Reichenheim, M. E., Verly Junior, E., Marques, E. S., \& Costa, R. S. (2015). Efeito da violência física entre parceiros íntimos no índice de massa corporal em mulheres adultas de uma população de baixa renda. Caderno de Saúde Pública, 31(1), 1-13.

Gomes, N. P., Erdmann, A. L., Stulp, K. P., Diniz, N. M. F., Correia, C. M., \& Andrade, S. R. (2014). Cuidado às mulheres em situação de violência conjugal: importância do 
psicólogo na Estratégia de Saúde da

Família. Psicologia USP, 25(1), 63-69.

Gomes, N. P., Garcia, T. C. S., Conceição, C. R., Sampaio, P. O., Almeida, V. C., \& Paixão, G. P. N. (2012). Violência conjugal: elementos que favorecem $\mathrm{O}$ reconhecimento do agravo. Saúde em Debate, 36(95), 514-522.

Idoko, P., Ogbe, E., Jallow, O., \& Ocheke, A. (2015). Burden of intimate partner violence in The Gambia: a cross sectional study of pregnant women. Reproductive Health, 12(34), 2-6.

Lettiere, A., \& Nakano, A. M. S. (2011). Violência doméstica: as possibilidades e os limites de enfrentamento. Revista Latino Americana de Enfermagem, 19(6), 1-8.

Lucena, K. D. T., Deininger, L. D. S. C., Coelho, H. F. C., Monteiro, A. C. C., de Toledo Vianna, R. P., \& do Nascimento, J. A. (2016). Analysis of the cycle of domestic violence against women. Journal of Human Growth and Development, 26(2), 139-146.

Ludermir, A. B., Valongueiro, S., \& Araújo, T. V. B. (2014). Common mental disorders and intimate partner violence in pregnancy. Revista de Saúde Pública, 48(1), 29-35.

Mendonça, M. F. S., \& Ludermir, A. B. (2017). Violência por parceiro íntimo e incidência de transtorno mental comum. Revista de Saúde Pública, 51(32), 1-8.

Netto, L. A., Moura, M. A. V., Queiroz, A. B. A., Tyrrell, M. A. R., \& Bravo, M. M. P. (2014). Violência contra a mulher e suas consequências. Acta Paulista Enfermagem, 27(5), 458-64.

Oliveira, A. R., \& D’Oliveira, A. F. P. L. (2008). Violência de gênero contra trabalhadoras de enfermagem em hospital geral de São Paulo (SP). Revista de Saúde Pública, 42(5), 868-876.

Oliveira, C. A., \& Almeida, L. C. G. (2013). HIV/AIDS e violência: situações de vulnerabilidade entre as mulheres. Revista Baiana de Saúde Pública, 37(4), 1029-1041.

Oliveira, P. P., Viegas, S. M. F., Santos, W. J., Silveira, E. A. A., \& Elias, S. C. (2015). Mulheres vítimas de violência doméstica: uma abordagem fenomenológica. Texto Contexto Enfermagem, 24(1), 196-203.

Parente, E. O., Nascimento, R. O., \& Vieira, L. J. E. S. (2009). Enfrentamento da violência doméstica por um grupo de mulheres após a denúncia. Estudos Feministas, 17(2), 445465.

Powell, V. B., Abreu, N., Oliveira, I. R., \& Sudak, D. (2008). Terapia cognitivocomportamental da depressão. Revista Brasileira de Psiquiatria, 30(2), 73-80.

Rodrigues, D. T., \& Nakano, A. M. S. (2007). Violência doméstica e abuso de drogas na gestação. Revista Brasileira de Enfermagem, 60(1), 77-80.

Santi, L. N., Nakano, A. M. S., \& Lettiere, A. (2010). Percepção de mulheres em situação de violência sobre o suporte e o apoio recebido em seu contexto social. Texto Contexto Enfermagem, 19(3), 417-424.

Schraiber, L. B., Barros, C. R. S., \& Castilho, E. A. (2010). Violência contra as mulheres por parceiros íntimos: usos de serviços de saúde. Revista Brasileira Epidemiol, 13(2), 237-245.

Schraiber, L. B., Latorre, M. R. D., França, I., Segri, N. J., \& D’Oliveira, A. F. P. L. (2010). Validade do instrumento Who Vaw Study 
para estimar violência de gênero contra a mulher. Revista de Saúde Pública, 44(4), 65866.

Silva, E. P., Valongueiro, S., Araújo, T. V. B., \& Ludermir, A. B. (2015). Incidência e fatores de risco para violência por parceiro íntimo no período pós-parto. Revista de Saúde Pública, 49(46), 1-9.

Silva, S. A., Lucena, K. D. T., Deininger, L. S. C., Coelho, H. F. C., Vianna, R. P. T., \& Anjos, U. U. (2015). Análise da violência doméstica na saúde das mulheres. Journal of Human Growth and Development, 25(2), 182186.

Vieira, L. B., Cortes, L. F., Padoin, S. M. M., Souza, I. E. O., Paula, C. C., \& Terra, M. G. (2014). Abuso de álcool e drogas e violência contra as mulheres: denúncias de vividos. Revista Brasileira de Enfermagem, 67(3), 366-372.

Waiselfisz, J. J. (2015). Mapa da violência 2015: homicídio de mulheres no Brasil. Brasília: Flacso.

Recebido em: 13/12/2017

Aprovado em: 21/11/2019 